\title{
La construction identitaire de l'enseignant dans son rapport à la littérature : apports d'une comparaison internationale
}

Dominique Ulma

\section{(2) OpenEdition \\ Journals \\ Édition électronique \\ URL : http://journals.openedition.org/trema/2532 \\ DOI : $10.4000 /$ trema.2532 \\ ISSN : 2107-0997 \\ Éditeur \\ Faculté d'Éducation de l'université de Montpellier}

Édition imprimée

Date de publication : 1 décembre 2010

Pagination : 44 - 53

ISSN : $1167-315 X$

\section{Référence électronique}

Dominique Ulma, «La construction identitaire de l'enseignant dans son rapport à la littérature apports d'une comparaison internationale », Tréma [En ligne], 33 - 34 | 2010, mis en ligne le 01 décembre 2012, consulté le 19 avril 2019. URL : http://journals.openedition.org/trema/2532 ; DOI : 10.4000/trema.2532

Ce document a été généré automatiquement le 19 avril 2019.

Trema 
La construction identitaire de l'enseignant dans son rapport à la littérature : apports d'une comparaison internationale

Dominique Ulma

I. Introduction : présentation de l'enquête 
1 L'International Mother tongue Education Network (IMEN), réseau européen informel de chercheurs en didactique de la langue de scolarisation (LSCo), travaille depuis 2007 à un projet de recherche sur le canon dans l'enseignement de la littérature, à la fois dans chacun des pays participants ${ }^{1}$ et dans une perspective comparative. Les didacticiens sont en effet, nationalement, confrontés à des questions similaires: explorer les différents modes de réponse grâce à une comparaison internationale permet de faire avancer la connaissance.

La recherche «Canon» d'IMEN prend appui sur un questionnaire soumis à des étudiants se destinant au professorat ${ }^{2}$, durant leur formation initiale. Le postulat de départ est que les attitudes des futurs enseignants face à la culture et à la

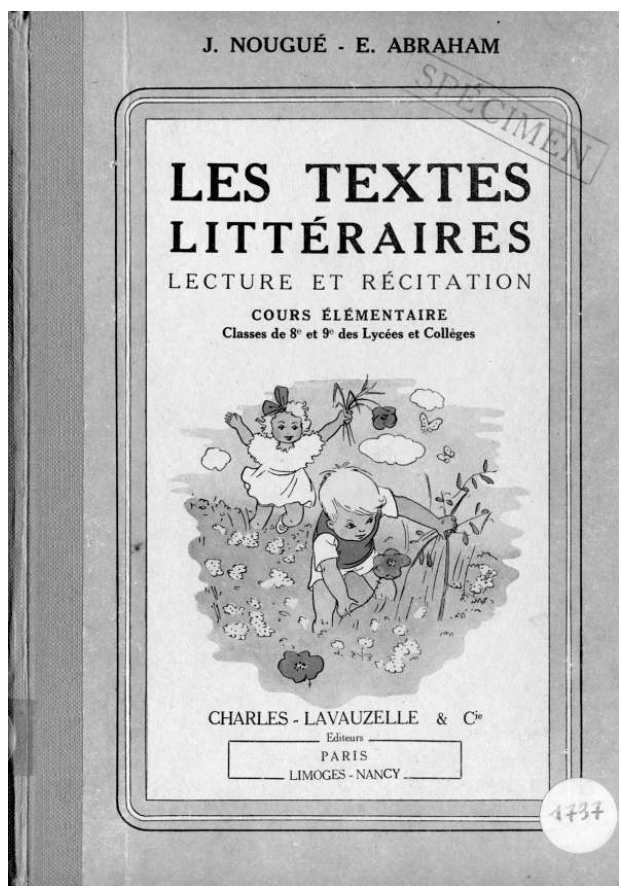
littérature sont déterminantes pour leur exercice futur du métier, qu'elles influent sur leurs prises de décisions. Il existe déjà des recherches en France (DEMOUGIN et MASSOL 1999 ; LUSETTI et QUET 2009), en Allemagne (Graf 2004), aux Pays-Bas (VERBOORD 2005) sur l'influence des lectures scolaires sur les pratiques de lecture privées et les pratiques professionnelles postulées : toutes, dans leur contexte national, montrent que la médiation est susceptible d'influencer la constitution du sujet lecteur. Qu'en est-il au niveau de la constitution d'une identité professionnelle d'enseignant?

La cible de l'enquête se caractérise par la conjonction de deux leviers de construction identitaire chez ces étudiants. D'une part, leur récente expérience d'élèves a contribué à construire leurs attitudes de lecture personnelles et leurs théories subjectives du bon cours de littérature. D'autre part, en tant qu'enseignants débutants, ils ont déjà des représentations voire des pratiques de médiateurs de littérature.

Les buts de l'enquête sont de repérer des profils de lecteurs et de vérifier si l'on peut corréler enseignement vécu, pratiques de lectures privées et identification à un type d'enseignement. C'est-à-dire les relations entre les pratiques de lecture privée et l'expérience de lecture scolaire des informateurs, les relations entre leurs pratiques de lecture privée et leur projection dans leur pratique future, les relations entre leur expérience de lecture scolaire et leur projection dans leur pratique future. La comparaison internationale permet de savoir si les corrélations ainsi mises à jour sont spécifiques à un pays ou transversales.

\section{Méthodologie et échantillon}

2 La méthodologie repose sur l'utilisation d'un questionnaire élaboré par l'équipe allemande $^{3}$, adapté pour le groupe IMEN, traduit en français, roumain, anglais et transposé sous une forme réduite en Norvège et Suède. Ce questionnaire comporte quelques données sociologiques et soixante questions (de type échelle et quelques 
questions ouvertes) centrées sur les pratiques culturelles et les pratiques de lecture littéraire ${ }^{4}$ actuelles des informateurs, leurs souvenirs scolaires, et il leur demande de se projeter dans la pratique de leur futur métier. Le traitement statistique des données allemandes, françaises et roumaines a été effectué par l'équipe allemande avec le logiciel SPSS. L'interprétation des données extraites fait l'objet non seulement d'un travail dans chaque pays mais aussi d'une élaboration comparée ${ }^{5}$. La procédure d'exploitation statistique adoptée relève de l'analyse factorielle exploratoire : tous les items traduisant un comportement de réponse inter corrélé sont regroupés dans un facteur commun (ou profil) qui correspond à une variable latente. Chaque variable latente est reconstruite par le chercheur par interprétation des éléments constitutifs du facteur. Pour tester la fiabilité, l'indice de consistance retenu est l'alpha de CRONBACH.

L'échantillon se compose de 544 questionnaires : 104 recueillis en France (IUFM de Lyon, Grenoble et Lille), 104 en Allemagne (faculté d'éducation d'Heidelberg et universités de Karlsruhe et Iena), 64 en Roumanie (universités de Bucarest et Brasov) ; la Norvège et la Suède ont recueilli 136 questionnaires chacune. Les caractéristiques d'âge et de genre des participants sont conformes aux statistiques générales dans les institutions de formation des différents pays et en Europe. Concernant la filière suivie, la comparaison n'est pas aisée dans la mesure où les systèmes éducatifs sont différents du point de vue de la structure des études, toutefois il ressort que, tous pays confondus, $77 \%$ des participants se destinent au primaire ou secondaire inférieur.

Malgré des défauts tenant en partie à la méthode choisie, en partie à la complexité de l'objet d'étude lui-même, les valeurs mises en évidence autorisent à formuler un certain nombre d'hypothèses fondées quant à l'attitude de ces futurs enseignants face à la lecture littéraire et aux éléments constitutifs de l'élaboration de leur identité professionnelle ${ }^{6}$.

\section{Les profils de lecteurs}

3 Sept profils de lecteurs ont pu être dégagés chez les étudiants allemands.

Le profil 1 correspond à la lecture esthétique. Ce sont des étudiants qui considèrent la littérature comme un art, s'intéressent à la langue des textes, aux intentions de l'auteur. Ce profil est toutefois peu représenté (moy. 2,227).

Le profil 2, la lecture - épanouissement, l'est un peu mieux (moy. 2,78): c'est celui d'étudiants dont la vie intérieure est nourrie par la lecture de textes littéraires.

Les étudiants du profil 3, la lecture légitimée, reconnaissent et acceptent la lecture de littérature comme pratique de formation et comme activité socialement valorisée. C'est le profil le mieux représenté (moy. 3,32).

Le profil 4, la lecture liée à des besoins, rassemble des étudiants qui lisent de la littérature pour satisfaire les besoins du moment, y compris des besoins personnels. Approuvé à peu près à la même hauteur (moy. 2,6), il est à la fois proche et différent du profil 5 de la lecture stratégique (moy. 2,65) qui correspond à des lecteurs développant des stratégies de lecture dans une attitude largement pragmatique et orientée vers des buts spécifiques.

Les étudiants du profil 6, la lecture - évasion, prennent peu de distance avec le texte dans lequel ils s'immergent; l'identification leur procure des émotions et stimule leur imagination. Ce profil recueille une forte approbation (moy. 3,17).

Le profil 7 renvoie à la lecture intéressée : ces étudiants, qui ne sont pas les plus nombreux (moy. 2,62), sont les plus pragmatiques de tous. Ils lisent principalement pour les besoins 
de leurs études mais aussi des textes qui font l'actualité : ils sont doublement influencés, par les nécessités de leurs études et par la sphère sociale.

Six profils de lecteurs ont été mis en évidence pour l'échantillon français.

Les lecteurs du profil 1, la lecture - fusion, s'impliquent émotionnellement dans la lecture, s'immergent dans le texte, lisent volontiers des classiques pour leurs loisirs et pour qui la littérature comporte des valeurs propres. Ce profil est le mieux représenté des six (moy. $3,01)$.

Le profil 2 de la lecture ciblée est le profil le moins bien représenté (moy. 2,17). Il s'agit de lecteurs pratiquant une sélection de leur lectures en fonction d'intérêts personnels, qui ne manifestent pas de goût particulier pour la littérature et ni pour la lecture en général.

Le profil 3, la lecture utilitaire (moy. 2,45), correspond à des étudiants qui eux aussi sélectionnent scrupuleusement leurs lectures, mais pour les besoins de leurs études, et attachent beaucoup de soin à rentabiliser cette activité, chez eux très cérébrale, débarrassée des émotions, au contraire du profil 1.

Le profil 4, la lecture centrée sur le personnage, regroupe des étudiants pour qui lire de la littérature, c'est d'abord s'attacher aux personnages, mais pas seulement dans un rapport de fusion : ils veulent aussi en comprendre les rouages. La lecture littéraire occupe une place importante dans leurs études. C'est un profil assez bien représenté (moy. 2,6).

Le lecteur-type du profil 5, la lecture - actualité (moy. 2,27), est avant tout en phase avec l'actualité : il lit les critiques, s'informe sur les dernières sorties et lit principalement des contemporains qu'il choisit en fonction de ses intérêts du moment, entretenant une certaine distance avec le texte.

Le profil 6, la lecture - épanouissement, le deuxième en importance (moy. 2,68), rassemble des lecteurs fortement impliqués dans la lecture, qui est pour eux un élément essentiel de développement personnel. Ils ritualisent l'acte de lire, moment intime et privilégié.

Les liens entre la filière et les profils font apparaître des différences selon le type d'établissement visé. On constate ainsi qu'en Allemagne, les étudiants se destinant au lycée manifestent une forte adhésion à la lecture esthétique et à la lecture épanouissement et une faible adhésion à la lecture liée à des besoins, tandis que ceux se destinant aux écoles primaires et élémentaires manifestent une faible adhésion à la lecture légitimée et une forte adhésion à la lecture intéressée, ce qui correspond à la représentation que l'on peut se faire des motivations à la lecture de ces étudiants. Les futurs enseignants en école élémentaire et au collège approuvent fortement la lecture intéressée, la lecture liée à des besoins et la lecture - évasion. Il est ainsi frappant d'observer que la lecture littéraire est majoritairement une activité imposée chez les étudiants se destinant au primaire et au secondaire inférieur.

Chez les participants français, la lecture - fusion et la lecture ciblée ne sont significativement représentées que chez les stagiaires professeurs des écoles (PE2). Dans les deux cas, l'intérêt personnel, émotionnel et intellectuel, prime. Ces mêmes stagiaires rejettent au contraire massivement la lecture centrée sur le personnage. La lecture épanouissement n'est significative que chez les étudiants se destinant au primaire (PE1). Est-ce à dire qu'une fois le concours en poche, soit les stagiaires trouvent moins le temps de lire, soit leurs préoccupations changent, et avec elles leur rapport à la lecture de littérature ? La lecture utilitaire et la lecture - actualité ne sont significatives d'aucune filière. Or, on aurait pu s'attendre à ce que les étudiants pratiquent davantage une lecture utilitaire, pour la préparation du concours. On aurait pu s'attendre aussi à ce qu'un ou plusieurs profils - lecture - fusion ou lecture - épanouissement - se distinguent chez ceux 
se destinant au secondaire : la faiblesse de l'échantillon et sa constitution expliquent peut-être ce résultat.

7 A la comparaison, deux attitudes majeures se dessinent : une lecture de littérature pour soi et une lecture ciblée en fonction d'intérêts extérieurs variés (réussir ses études, garder le contact avec l'actualité). Mais on note aussi une bipolarisation autour des bénéfices que le lecteur souhaite tirer de sa lecture, entre une lecture pour la satisfaction intellectuelle et une lecture pour la satisfaction émotionnelle.

\section{L'expérience scolaire}

8 Les expériences personnelles vécues en classe de LSco permettent de mettre en évidence les modèles d'enseignement qu'ont connus les informateurs Ainsi, on repère trois modèles chez les étudiants allemands, le premier qui renvoie au cours de littérature considéré comme partie intégrante de la vie littéraire (moy. 2,36), le deuxième au cours de littérature axé sur les pédagogies actives (moy. 2,64) et le troisième au cours de littérature comme lieu de transmission de savoirs (moy. 3,01).

Il n'y a pas de corrélation visible entre pratiques de lecture privées et modèles d'enseignement, mais en ce qui concerne la filière d'études, on note une faible représentation de tous les modèles chez les étudiants se destinant au primaire et à l'élémentaire: à moins qu'ils n'aient aucun souvenir de leur passé d'écoliers, l'enseignement reçu n'a pas produit chez eux d'impact significatif. En revanche, les étudiants se destinant au lycée approuvent de manière supérieure à la moyenne le modèle du cours de littérature comme faisant partie de la vie littéraire, alors que ceux se destinant à l'école élémentaire et au collège privilégient le modèle axé sur les pédagogies actives. Il semble donc qu'il y ait chez eux un lien entre l'expérience scolaire et la filière d'études choisie.

9 Les réponses des participants français sur leur expérience d'écoliers permettent de ne dégager que deux modèles didactiques : un modèle plutôt traditionnel, où le professeur orchestre le savoir dont il assure la transmission. Ce modèle, le plus fréquent (moy. 2,56), est mentionné par toutes les catégories d'étudiants, avec une représentation plus forte chez les PE1 et plus faible chez les PE2. Et un modèle davantage versé dans les pédagogies actives où l'élève est mis en avant. Ce modèle, beaucoup moins fréquent (moy. 1,85), est fort chez les PE1, et sous-représenté chez les PE2 et tous les informateurs du second degré. On peut donc penser que le modèle d'enseignement reçu n'a pas d'influence sur le choix de carrière des PE1, alors que ceux se destinant au secondaire semblent avoir peu l'expérience des pédagogies actives, sans qu'un modèle soit plus prescripteur que l'autre. L'influence de l'enseignement reçu sur les profils de lecteurs n'est pas fortement marquée, mais on observe quelques tendances: l'enseignement traditionnel a plutôt formé des lecteurs qui ont un rapport utilitaire à la lecture; les méthodes actives ont plutôt formé des lecteurs qui pratiquent la lecture ciblée, se focalisent sur le personnage ou l'actualité, et ceux pour qui la lecture littéraire relève du développement personnel.

En somme, on peut dire que si l'on veut que les élèves lisent de la littérature plus tard, les pédagogies actives, centrées sur l'élève, son activité, ses centres d'intérêt, ont plus de chances de se révéler efficaces. Toutefois les corrélations ne sont pas assez significatives et le modèle des pédagogies actives est trop peu présent dans les souvenirs des étudiants pour que l'on puisse affirmer qu'il y a un impact déterminant. Une combinaison de facteurs est plus probable. 
10 En France et en Allemagne, les étudiants se destinant au secondaire ont plutôt reçu un enseignement de type traditionnel tandis que ceux se destinant au primaire ont davantage connu les méthodes actives.

\section{La projection dans le métier}

11 Par la mise en évidence des critères privilégiés pour le choix des œuvres littéraires à faire étudier en classe, on repère quatre types d'enseignants en devenir chez les étudiants allemands : ceux qui privilégient les intérêts et besoins des élèves; ceux qui privilégient les injonctions scolaires (programmes, examens, moy. 3,06); ceux qui privilégient la faisabilité pratique en classe; ceux qui privilégient les intérêts et préférences de l'enseignant.

Les étudiants se destinant au primaire et à l'école élémentaire manifestent un consentement supérieur à la moyenne aux intérêts des élèves et aux préférences de l'enseignant, et inférieur à la moyenne aux deux autres facteurs. Ils semblent ainsi se préoccuper avant tout des besoins des acteurs présents dans la classe, élèves et maître. Les étudiants se destinant à l'école élémentaire et au collège n'ont pas de préférence marquée pour les types 1,3 et 4 mais ils rejettent le type 2 des injonctions scolaires : cela pourrait signifier que ces étudiants, qui doivent s'adapter à des élèves au niveau généralement faible, ont une vision pragmatique articulée avec les réalités contingentes du métier. Les étudiants se destinant au lycée n'ont pas de préférence marquée pour les types 1, 2 et 3 mais ils rejettent le type 4 (préférences de l'enseignant), ce qui semble montrer une prise en compte de tous les facteurs d'influence dans la compréhension des textes.

12 Chez les étudiants français se dessinent trois types d'enseignants en devenir. L'enseignant de type 1 a pour préoccupation première l'intérêt des élèves, dans le respect des programmes. Il est très largement représenté (moy. 3,54) et est similaire au type 1 des étudiants allemands, lui aussi très significatif (moy. 3,57). Ce type correspond davantage aux étudiants se destinant à devenir professeurs des écoles alors qu'il est peu représenté chez ceux se destinant au secondaire, aspect partagé avec les étudiants allemands.

L'enseignant de type 2 se fie beaucoup à sa propre expérience, d'élève et de lecture privée, et s'appuie sur les programmes d'examen pour effectuer sa sélection. L'impact d'une forme de reproduction est ici beaucoup plus fort. C'est le type le plus faiblement représenté des trois (moy. 2,52). On peut le rapprocher du type 4 des étudiants allemands, mais en moins significatif (moy. 2,86). Ce type se retrouve chez les étudiants se destinant au premier comme au second degré, mais est rejeté par les PE2 : la confrontation au réel par la pratique de classe aurait-elle un effet sur les choix ?

L'enseignant de type 3 se préoccupe d'abord de la faisabilité en classe et du caractère d'exemplarité des œuvres étudiées. Ce type se retrouve fréquemment (moy. 3,28) et renvoie au type 3 des étudiants allemands (moy. 3,08). Il est bien représenté chez les futurs professeurs des écoles et ceux des lycées professionnels, mais pas chez les futurs documentalistes, peut-être parce que ceux-ci n'ont pas autant que les professeurs le souci de la mise en œuvre didactique.

13 Le choix d'une œuvre pour l'étude en classe ne peut dépendre que d'un seul facteur, de sorte que les types 1 et 3 sont souvent combinés, c'est-à-dire que la plupart des étudiants interrogés souhaitent avant tout proposer des œuvres qui satisfassent leurs futurs élèves, mais ils veulent aussi que ce choix leur garantisse une bonne opérationnalité pratique. Ils 
ne chercheront pas à se mettre en difficulté professionnellement parlant juste pour faire plaisir à leurs élèves. Leurs choix sont par conséquent mûrement réfléchis et négociés, et tiennent en plus compte des programmes. On peut faire l'hypothèse qu'il s'agit là d'une compétence professionnelle en cours d'élaboration.

Toujours chez les Français, on note des corrélations entre les profils de lecteurs et la projection dans des types d'enseignement. Chez les étudiants dont les choix sont motivés avant tout par les intérêts et goûts des élèves, les pratiques de lecture privée reposent elles aussi sur l'intérêt personnel et le goût pour la littérature : leurs motivations à la transmission d'un patrimoine littéraire ne se fondent donc pas sur la qualité intrinsèque des textes, mais sur le rapport aux textes que le lecteur peut instaurer.

Chez ceux qui ont une visée plus pragmatique du choix des textes littéraires, on relève assez logiquement une forte corrélation avec les profils de la lecture utilitaire et de la lecture ciblée. Ces étudiants, qui ne se préoccupent pas au premier chef d'épanouissement personnel, sont soucieux de transmettre une culture littéraire exemplaire, quitte à ce que celle-ci le soit au détriment d'un rapport plus intime au texte. On peut s'étonner que le type 2, qui repose sur un lien fort entre l'expérience personnelle de l'étudiant et sa projection dans le métier, ne soit pas plus nettement représenté chez les étudiants chez qui la lecture de littérature apporte des satisfactions sur le plan personnel: faut-il en conclure que ces étudiants se sentent encore eux-mêmes très «élèves » et pas encore réellement professeurs, et ainsi ne se projettent pas encore dans le métier? Toute construction identitaire est progressive.

Chez les étudiants allemands, on note aussi des corrélations. Ceux qui privilégient les préférences de l'enseignant montrent une forte adhésion à la lecture liée à des besoins et en même temps une faible adhésion à la lecture esthétique : si les futurs enseignants qui choisissent les textes sur la base de leurs préférences personnelles n'ont pas de goût pour les qualités esthétiques, des textes, cela sonne-t-il la fin de la «formation littéraire humaniste " (Bildung)? A l'inverse, les étudiants qui préfèrent la lecture esthétique veulent privilégier les textes, leur exemplarité et la faisabilité de leur étude: eux perpétuent le modèle d'enseignement tourné vers la Bildung, mais ils sont loin d'être majoritaires dans l'échantillon.

Les étudiants qui pratiquent la lecture - épanouissement ont des affinités avec le type d'enseignant qui privilégie la centration sur les textes et leur faisabilité et celui qui vise les intérêts de l'élève. Lire la littérature pour des motifs pédagogiques est une finalité bien acceptée en Allemagne: s'appuyer sur ses goûts personnels est peut-être un bon moyen de satisfaire par la même occasion des buts pédagogiques.

Enfin, si l'on cherche des corrélations entre l'enseignement reçu et les pratiques d'enseignement projetées, les étudiants qui ont été enseignés de manière traditionnelle (France) et ceux dont les cours visaient avant tout la transmission de savoirs (Allemagne) ont plutôt tendance à privilégier l'intérêt et les besoins des élèves, sans doute pour compenser le fait de n'avoir pas assez été acteurs lors des cours de littérature, dont ils gardent des souvenirs relativement inconfortables des deux côtés du Rhin. Les Français s'appuient aussi sur les programmes et leur expérience personnelle, faute de références peut-être.

Quant aux Français qui ont connu à l'école des pédagogies actives, ils privilégient certes l'intérêt des élèves, mais moins que la faisabilité en classe: n'ayant pas encore fait l'expérience de ces pédagogies, sont-ils impressionnés par la complexité de leur mise en 
oeuvre en classe ? Ou au contraire considèrent-ils que ces démarches sont à même de les aider dans l'organisation de l'étude de textes littéraires?

\section{Conclusion}

Les étudiants qui se destinent au professorat en Europe et qui auront à enseigner la littérature de la LSco sont des lecteurs: c'est rassurant. Mais ce ne sont pas, sauf exception, de gros lecteurs de littérature pour leurs loisirs. Leurs pratiques de lecture sont variées, se retrouvent de manière transversale et sont moins influencées par l'enseignement qu'ils ont reçu que par des facteurs contemporains: l'actualité et les études entreprises.

Il semblerait en revanche que l'expérience scolaire ait eu une influence d'une part sur le choix de leur filière d'étude - primaire ou secondaire en France, élémentaire ou filière d'élite du Gymnasium en Allemagne -, d'autre part sur l'image du métier qu'ils se font mais plus comme repoussoir que comme modèle - rejet du modèle traditionnel dans le souci de ne pas reproduire de mauvaises expériences.

A ce stade, nous disposons de profils de lecteurs, de modèles d'enseignement reçus et de types d'enseignants en projection : ces données, complétées des questionnaires roumains et belges, doivent permettre de fabriquer un nouveau questionnaire ciblant les facteurs transversaux aux pays, questionnaire qui pourra être soumis sur une cohorte plus importante et ciblée quantitativement en fonction des filières, pour davantage de représentativité et vérifier les tendances dégagées.

\section{BIBLIOGRAPHIE}

DEMOUGIN P. et MASSOL J.-F. (COORD.). 1999. Lecture privée et lecture scolaire, la question de la littérature à l'École, Actes de la journée d'étude de l'IUFM de Nîmes. Grenoble : CRDP de Grenoble, Coll. Documents, actes et rapports

GRAF W. 2004. DER SINN DES LESENS. Modi der literarischen Rezeptionskompetenz. Münster : LIT LUSETTI M. et QUET F. (2009). « Avenirs des lectures scolaires », in Daunay B., Delcambre I., Reuter Y. (éds), Didactique du français : le socioculturel en question. Lille : Presses Universitaires du Septentrion, Coll. Éducation et didactiques. 123-136

PIEPER I., AASE L., SÂMIHĂIAN F., FLEMING M. 2007. Texte, littérature et "Bildung" : Perspectives comparées .Strasbourg : Conseil de l'Europe.

ULMA D., (à paraitre), « "Du corpus scolaire à la bibliothèque intérieure" : vers une comparaison internationale », in Rouxel Annie et Louichon Brigitte (dir.), Actes de la 9ème rencontre des chercheurs en didactique de la littérature Du corpus scolaire à la bibliothèque intérieure, IUFM de Bordeaux, 3-5 avril 2008. Presses Universitaires de Rennes

ULMA D., (à paraitre), « How do French student teachers deal with literature and its teaching: canon between curriculum and personal preferences ", Actes du colloque "National Literatures in 
the age of Globalization: the issue of the Canon", Université de Bucarest, Roumanie, 31 oct-1er nov 2008

VERBOORD M. 2005. « Long-term effects of literary education on book-reading frequency: An analysis of Dutch student cohorts 1975-1998 ». in JANSSEN S. et PETERSON R. A. (éds.). Poetics, vol. $33, \mathrm{n}^{\circ} 5-6$, Comparative research on cultural production and consumption. Amsterdam : Elsevier. $320-342$

\section{NOTES}

1. Norvège, Suède, Roumanie, Allemagne, Belgique et France

2. Ils enseigneront forcément la LSco et sa littérature : dans le cadre de la polyvalence dans le premier degré, comme matière privilégiée ou unique dans le second degré

3. Dans le cadre du projet "Culture littéraire" de B. Rank et Ch. Bräuer à la faculté de pédagogie d'Heidelberg (<http://www.ph-heidelberg.de/wp/haerle/litbild.htm>)

4. Au sens de lecture de textes littéraires (Dufays 2004)

5. Intervention avec Iris Winkler, "L'enquête IMEN-Littérature : vers une comparaison francoallemande du rapport des futurs enseignants au canon littéraire ", Journée d'études francoallemande du Centre interdisciplinaire d'études et de recherches sur l'Allemagne (CIERA), Université Louis Lumière Lyon 2, "Canon scolaire et pratiques d'enseignement", IUFM, Lyon, 9 juin 2009

6. On ne retiendra ici que les données françaises et allemandes, qui offrent la plus large base de comparaison

7. Les valeurs moyennes d'approbation des différents facteurs peuvent mathématiquement se situer entre 1 (faible) et 4 (fort)

\section{RÉSUMÉS}

Malgré leurs différences quant aux corpus à enseigner, aux finalités et aux modalités d'enseignement, les pays européens ont en partage de faire travailler les élèves sur des objets littéraires, au primaire comme au secondaire. En matière de littérature, l'enseignant est aussi, et avant même d'être un enseignant, un lecteur. Comment se construit-il une identité d'enseignant de littérature, dans l'articulation de son passé d'ancien élève, de ses rencontres privées avec la littérature, des effets de la formation et des prescriptions des programmes?

Si l'on dispose de données sur le contexte francophone, il existe peu d'études comparatives au niveau international. Comment se fait cette alchimie dans un autre système éducatif, dans un autre panorama littéraire? Quels sont les principaux facteurs d'influence ? Peut-on identifier des transversalités au niveau européen?

Nous appuyant sur une enquête européenne, nous montrerons, dans une perspective comparée, les profils de lecteurs des étudiants et analyserons les corrélations entre ces profils et leurs pratiques de lecture, leurs préférences, leur expérience d'écolier et leurs postures professionnelles en devenir. 
Despite differences in the canon, aims and teaching methods, in all European countries students work on literary objects, both in elementary and secondary schools. As far as literature is concerned, the teacher is also, and even before being a teacher, a reader. How is his/her identity of a literature teacher being built, taking into account altogether his/her experience as a student, his/her private encounters with literature, the effects of teacher training and the curriculum requirements?

If data are available on the French context, there are few international comparative studies. How does the alchemy works in another educational system, in another literary scene? Which are the main influences? Can we identify transversal issues at a European level?

Relying on a European survey, we will point out, in a comparative perspective, the reading profiles of the students and will analyze correlations between these profiles and their reading habits, their preferences, their experience as a pupil and their future professional choices.

INDEX

Mots-clés : comparaison internationale, enseignant, identité, littérature

\section{AUTEUR}

DOMINIQUE ULMA

IUFM de l'académie de Lyon - Université Lyon I 